\title{
Anisotropic United Atom model including the electrostatic interactions of methyl-benzenes. II. Transport properties.
}

\author{
Carlos Nieto-Draghi, Patrick Bonnaud and Philippe Ungerer \\ IFP, 1-4 Avenue de Bois-Préau, 92852 Rueil-Malmaison, Cedex, France
}

Carlos.nieto@ifp.fr

\section{SUPPLEMENTAL INFORMATION}

Table A1: Temperature and pressure dependence of the self-diffusion coefficient D for toluene and xylene isomers. Reorientational relaxation times of $\mathrm{CH}$ vectors of hydrogen atoms in position ortho $(o)$, meta $(m)$ and para $(p)$ with respect to the methyl group and rotational diffusion constants $\mathrm{D}_{\mathrm{x}}, \mathrm{D}_{\mathrm{y}}$ and $\mathrm{D}_{\mathrm{z}}$ around the principal axes for Toluene at 0.1MPa. Percentage of deviation with respect the experimental data in parenthesis. Results for OPLS model of xylene isomers are taken from the work of Rousseau et al. ${ }^{64}$ and for the flexible toluene model from the work of Fioroni et al. ${ }^{65}$ Deviation from experimental data between parenthesis.

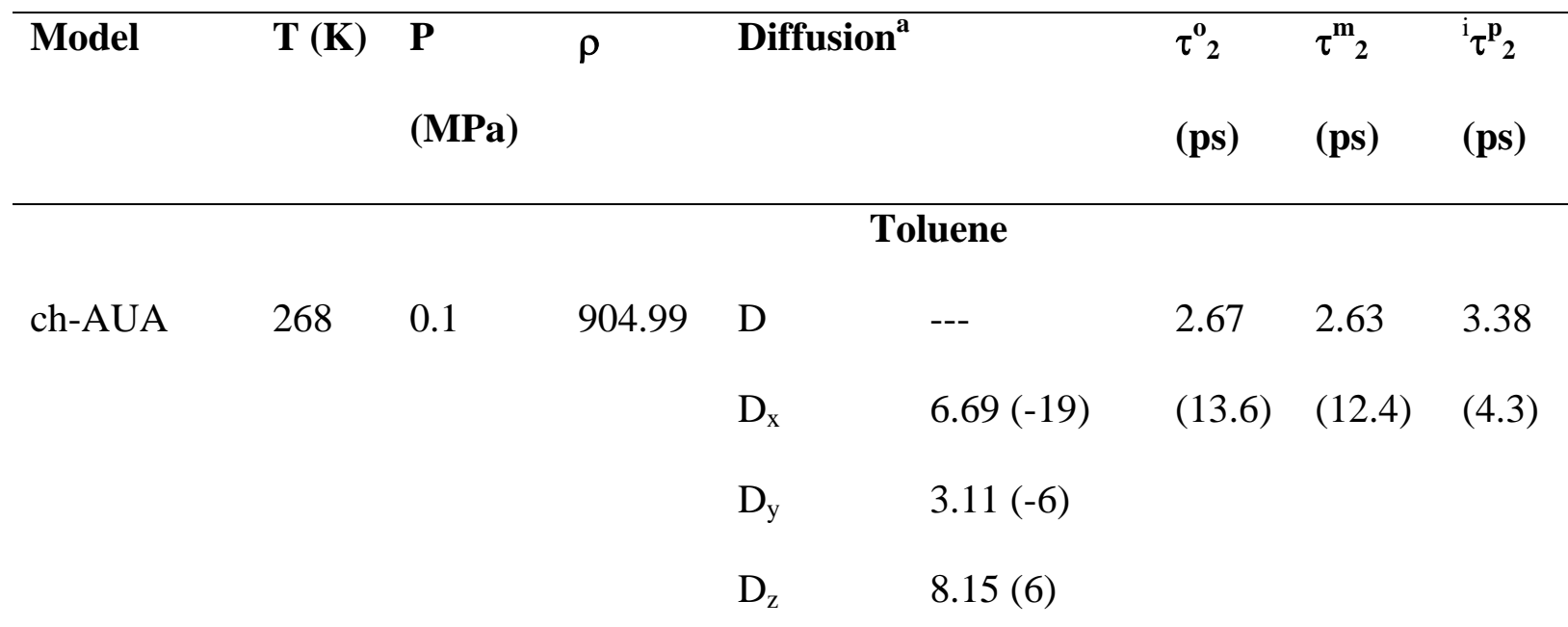


Exp.

$890.18^{\mathrm{b}} \quad \mathrm{D}$

$1.1^{\mathrm{d}}$

$\begin{array}{lll}2.35^{\mathrm{g}} \quad 2.34^{\mathrm{g}} \quad 3.24^{\mathrm{g}} & \end{array}$

$\begin{array}{ll}\mathrm{D}_{\mathrm{x}} & 8.31\end{array}$

$\mathrm{D}_{\mathrm{y}} \quad 3.30$

$\begin{array}{ll}\mathrm{D}_{\mathrm{z}} & 7.67\end{array}$

ch-A
Exp.

894.87 D

$\mathrm{D}_{\mathrm{x}} \quad 7.25(-17)$

$2.39 \quad 2.50 \quad 3.19$

$\mathrm{D}_{\mathrm{y}}$

$3.21(-17)$

$\mathrm{D}_{\mathrm{z}}$

$8.91(7)$

$\begin{array}{ccc}880.9^{\mathrm{b}} & \mathrm{D} & 1.6^{\mathrm{d}} \\ & \mathrm{D}_{\mathrm{x}} & 8.70 \\ & \mathrm{D}_{\mathrm{y}} & 3.87 \\ & \mathrm{D}_{\mathrm{z}} & 8.31\end{array}$

ch-AUA

$288 \quad 0.1$

885.95

D

$---$

$\begin{array}{lll}2.26 & 2.23 \quad 2.86\end{array}$

$\mathrm{D}_{\mathrm{X}}$

$6.94(17)$

(16.5) (16.1) (14.9)

$\mathrm{D}_{\mathrm{y}}$

$3.60(-8)$

$\mathrm{D}_{\mathrm{z}}$

$10.08(-4)$

Exp.

$871.67^{\mathrm{b}} \quad \mathrm{D}$

$1.9^{\mathrm{d}}$

$\begin{array}{lll}1.94^{\mathrm{g}} & 1.92^{\mathrm{g}} \quad 2.49^{\mathrm{g}}\end{array}$

$\begin{array}{ll}\mathrm{D}_{\mathrm{x}} & 8.41\end{array}$

$\begin{array}{ll}\mathrm{D}_{\mathrm{y}} & 3.92\end{array}$

$\begin{array}{ll}\mathrm{D}_{\mathrm{z}} & 10.56\end{array}$

ch-AUA 298

0.1

$876.64 \quad D$

$2.20 \pm 0.05\left(\begin{array}{llll}0 & 2.01 & 1.98 & 2.52\end{array}\right.$

$\mathrm{D}_{\mathrm{x}}$

(9.8) (8.8) (3.7)

$\mathrm{D}_{\mathrm{y}} \quad 8.8(-13)$

$\mathrm{D}_{\mathrm{z}} \quad 4.5(1)$

$12.45(25)$

Flexible

$864.1 \quad \mathrm{D}$

$2.6 \pm 0.05(18$

Exp.

$860.8^{\mathrm{c}} \quad \mathrm{D}$

$\begin{array}{llll}2.2^{\mathrm{d}} / 2.7^{\mathrm{f}} & 1.83^{\mathrm{g}} & 1.82^{\mathrm{g}} & 2.43^{\mathrm{g}}\end{array}$ 


\begin{tabular}{|c|c|c|c|c|c|c|c|c|}
\hline & & & & $\mathrm{D}_{\mathrm{x}}$ & 10.09 & & & \\
\hline & & & & $\mathrm{D}_{\mathrm{y}}$ & 4.44 & & & \\
\hline & & & & $\mathrm{D}_{\mathrm{z}}$ & 9.97 & & & \\
\hline ch-AUA & 313 & 0.1 & 863.02 & $\mathrm{D}$ & $2.62 \pm 0.07(-$ & -- & -- & -- \\
\hline & & & & & 3) & & & \\
\hline Exp & & & $846.1^{\mathrm{c}}$ & $\mathrm{D}$ & $2.70^{\mathrm{d}} / 3.1^{\mathrm{f}}$ & $1.57^{\mathrm{g}}$ & $1.57^{\mathrm{g}}$ & $2.01^{\mathrm{g}}$ \\
\hline & & & & $\mathrm{D}_{\mathrm{x}}$ & 10.32 & & & \\
\hline & & & & $\mathrm{D}_{\mathrm{y}}$ & 4.54 & & & \\
\hline & & & & $\mathrm{D}_{\mathrm{z}}$ & 11.83 & & & \\
\hline ch-AUA & 333 & 0.1 & 843.3 & ${ }^{\mathrm{c}} \mathrm{D}$ & $3.44 \pm 0.09(-$ & 1.42 & 1.45 & 1.94 \\
\hline & & & & $\mathrm{D}_{\mathrm{x}}$ & 3) & (7.6) & $(9.8)$ & (14.1) \\
\hline & & & & $\mathrm{D}_{\mathrm{y}}$ & 12.96 & & & \\
\hline & & & & $\mathrm{D}_{\mathrm{z}}$ & 5.98 & & & \\
\hline & & & & & 18.10 & & & \\
\hline Exp & & & $827.5^{c}$ & $\mathrm{D}$ & $3.54^{\mathrm{d}, \mathrm{e}} / 4.0^{\mathrm{f}}$ & $1.32^{\mathrm{g}, \mathrm{h}}$ & $1.32^{\mathrm{g}, \mathrm{h}}$ & $1.70^{\mathrm{g}, \mathrm{h}}$ \\
\hline & & & & $\mathrm{D}_{\mathrm{x}}$ & & & & \\
\hline & & & & $\mathrm{D}_{\mathrm{y}}$ & & & & \\
\hline & & & & $\mathrm{D}_{\mathrm{z}}$ & & & & \\
\hline ch-AUA & 353 & 0.1 & 823.53 & $\mathrm{D}$ & $4.12 \pm 0.11$ & -- & -- & -- \\
\hline & & & & & 9) & & & \\
\hline Exp. & & & $808.3^{c}$ & $\mathrm{D}$ & $4.52^{\mathrm{d}, \mathrm{e}} / 5.2^{\mathrm{f}}$ & $1.15^{\mathrm{g}, \mathrm{h}}$ & $1.15^{\mathrm{g}, \mathrm{h}}$ & $1.43^{\mathrm{g}, \mathrm{h}}$ \\
\hline ch-AUA & 363 & 0.1 & 814.7 & $\mathrm{D}$ & $4.55 \pm 0.12$ & 1.03 & 1.05 & 1.37 \\
\hline & & & & $\mathrm{D}_{\mathrm{x}}$ & 18.18 & & & \\
\hline & & & & $\mathrm{D}_{\mathrm{y}}$ & 7.58 & & & \\
\hline & & & & $\mathrm{D}_{\mathrm{z}}$ & 21.52 & & & \\
\hline Exp. & & & $798.7^{\mathrm{C}}$ & $\mathrm{D}$ & $5.7^{\mathrm{f}}$ & -- & -- & -- \\
\hline ch-AUA & 333 & 40.0 & 875.24 & ${ }^{\mathrm{c}} \mathrm{D}$ & $2.53 \pm 0.09$ & -- & -- & -- \\
\hline ch-AUA & 333 & 100.0 & 908.99 & $\mathrm{D}$ & $1.99 \pm 0.12$ & -- & -- & -- \\
\hline
\end{tabular}


$\begin{array}{lllll}\text { ch-AUA } & 333 \quad 500.0 & 1026.4 & \mathrm{D}\end{array}$

$0.45 \pm 0.13$

AAD (\%)

ch-AUA

D

3.8

$\tau_{2}^{\mathrm{o}} \quad 11.2$

$\mathrm{D}_{\mathrm{x}}$

16.5

$\tau_{2}^{\mathrm{m}} \quad 12.4$

$\mathrm{D}_{\mathrm{y}}$

8

$\tau_{2}^{\mathrm{p}_{2}} \quad 9.4$

$\mathrm{D}_{\mathrm{z}}$

10.5

o-xylene

$\tau^{\perp}{ }_{2} \quad \tau_{2=}^{\mathrm{o}} \tau^{\mathrm{y}}{ }_{2} \quad \tau^{\mu}{ }_{2}$

ch-AUA

273

0.1

988

D

0.97

OPLS

$298 \quad 0.1$

875.8

D

1.31

ch-AUA

878.9

D

1.51

$\begin{array}{lll}6.01 & 3.15 \quad 3.88\end{array}$

$\mathrm{D}_{\mathrm{x}} \quad 13.6$

$\begin{array}{ll}\mathrm{D}_{\mathrm{y}} & 8.4\end{array}$

$\begin{array}{ll}\mathrm{D}_{\mathrm{z}} & 9.8\end{array}$

OPLS

323

0.1

$853.1 \quad \mathrm{D}$

1.90

ch-AUA

855.8 D

2.02

OPLS

$348 \quad 0.1$

828.6

D

2.64

ch-AUA

833.4 D

2.81

OPLS

373

0.1

D

ch-AUA

809.9

D

3.35

OPLS

323

40.0

882.5 D

1.33

Ch-AUA

884.0

D

1.53

OPLS

323

100.0

914.3

D

0.94

Ch-AUA

$$
915.0
$$

D

1.08

m-xylene

$\tau^{\perp}{ }_{2} \quad \tau^{\mathrm{y}}{ }_{2} \quad \tau_{2=}^{\mathrm{m}} \tau_{2}^{\mu}$

Ch-AUA

273

0.1

890.0

D

1.28

OPLS

$298 \quad 0.1$

858.0

D

1.73 


\begin{tabular}{|c|c|c|c|c|c|c|c|c|}
\hline \multirow[t]{4}{*}{ Ch-AUA } & & & 868.0 & $\mathrm{D}$ & 1.87 & 5.33 & 5.41 & 3.91 \\
\hline & & & & $\mathrm{D}_{\mathrm{x}}$ & 9.66 & & & \\
\hline & & & & $\mathrm{D}_{\mathrm{y}}$ & 9.47 & & & \\
\hline & & & & $\mathrm{D}_{\mathrm{z}}$ & 9.55 & & & \\
\hline OPLS & 323 & 0.1 & 835.7 & $\mathrm{D}$ & 2.46 & & & \\
\hline Ch-AUA & & & 845.8 & $\mathrm{D}$ & 2.59 & & & \\
\hline OPLS & 348 & 0.1 & 810.9 & $\mathrm{D}$ & 3.30 & & & \\
\hline Ch-AUA & & & 823.0 & $\mathrm{D}$ & 3.51 & & & \\
\hline OPLS & 373 & 0.1 & -- & $\mathrm{D}$ & -- & & & \\
\hline Ch-AUA & & & 800.1 & $\mathrm{D}$ & 4.32 & & & \\
\hline OPLS & 323 & 40.0 & 865.4 & $\mathrm{D}$ & 1.81 & & & \\
\hline Ch-AUA & & & 873.9 & $\mathrm{D}$ & 1.96 & & & \\
\hline OPLS & 323 & 100.0 & 898.6 & $\mathrm{D}$ & 1.20 & & & \\
\hline \multirow[t]{2}{*}{ Ch-AUA } & & & 906.2 & $\mathrm{D}$ & 1.41 & & & \\
\hline & & & & & $p$-xylene & $\tau^{\perp}{ }_{2}$ & $\tau^{\mathrm{y}}{ }_{2}$ & ${ }^{\mathrm{j}} \tau^{\mathrm{x}}{ }_{2}$ \\
\hline OPLS & 298 & 0.1 & 854.0 & $\mathrm{D}$ & 1.91 & & & \\
\hline \multirow[t]{4}{*}{ Ch-AUA } & & & 865.0 & $\mathrm{D}$ & 2.06 & 4.46 & 3.15 & 5.83 \\
\hline & & & & $\mathrm{D}_{\mathrm{x}}$ & 28.2 & & & \\
\hline & & & & $\mathrm{D}_{\mathrm{y}}$ & 5.62 & & & \\
\hline & & & & $\mathrm{D}_{\mathrm{z}}$ & 4.97 & & & \\
\hline OPLS & 323 & 0.1 & 831.5 & $\mathrm{D}$ & 2.74 & & & \\
\hline Ch-AUA & & & 842.5 & $\mathrm{D}$ & 2.57 & & & \\
\hline OPLS & 348 & 0.1 & 807.3 & $\mathrm{D}$ & 3.60 & & & \\
\hline Ch-AUA & & & 819.5 & $\mathrm{D}$ & 3.51 & & & \\
\hline OPLS & 373 & 0.1 & -- & $\mathrm{D}$ & -- & & & \\
\hline Ch-AUA & & & 795.6 & $\mathrm{D}$ & 4.24 & & & \\
\hline OPLS & 323 & 40.0 & 862.01 & $\mathrm{D}$ & 1.98 & & & \\
\hline
\end{tabular}


a Translational self-diffusion coefficient D in $\mathrm{cm}^{2} / \mathrm{s}$, rotational diffusion coefficients in $10^{10} \mathrm{rad}^{2} / \mathrm{s}$. ${ }^{\mathrm{b}}$ Density from NIST database (http://webbook.nist.gov/chemistry). ${ }^{c}$ Experimental data from EtTahir. ${ }^{66}{ }^{\mathrm{d}}$ Experimental self-diffusion coefficient $\mathrm{D}$ from Krüger et al. ${ }^{60}$ e Extrapoled from the experimental data using the coefficients of reference $60, D=D_{0} * \exp \left(E^{D} / R T\right){ }^{f}$ Data interpolated form experimental self-diffusion coefficinet of Pickup et al. ${ }^{63}{ }^{\mathrm{g}}$ Experimental rotational diffusion coefficients $\left(D_{x}, D_{y}\right.$ and $\left.D_{z}\right)$ and relaxation times $\left(\tau_{0}^{2}, \tau_{m}^{2}\right.$ and $\left.\tau_{p}^{2}\right)$ from reference 46 . ${ }^{\text {h }}$ Extrapoled from the experimental data using coefficients of reference $46, \tau^{2}{ }_{\mathrm{i}}=\tau^{2}{ }_{0}{ }^{*} \exp \left(\mathrm{E}^{\tau} \mathrm{a} / \mathrm{RT}\right)$. ${ }^{\mathrm{i}}$ For toluene molecule, reorientational time $\tau_{p}^{2}=\tau_{\mu}^{2}$ due to the rigid character of the AUA model. ${ }^{\mathrm{j}}$ For p-xylene, $\tau_{x}^{2}$ corresponds to the reorientation of the vector joining the two methyl groups (see figure 1 for details). 
Table A2: Temperature and pressure dependence of the shear viscosity of alkyl-benzene computed for different models. Results for OPLS model of xylene isomers are taken from the work of Rousseau et al. ${ }^{\text {Error! Bookmark not defined. }}$ and for the flexible toluene model from the work of Fioroni et $a l$. Error! Bookmark not defined.

\begin{tabular}{|c|c|c|c|c|c|c|}
\hline Model & $\mathrm{T}(\mathrm{K})$ & $\mathrm{P}(\mathrm{Mpa})$ & $\rho(\mathrm{kg} / \mathrm{m} 3)$ & $\rho \%$ Dev & $\eta(10-6 P a . s)$ & $\eta \%$ Dev \\
\hline & & & Toluene & & & \\
\hline Ch-AUA & 298 & $0.1 \pm 0.4$ & $877.9+-0.5$ & 1.9 & $501 \pm 20$ & -10.7 \\
\hline Flexible & & 0.2 & 863.9 & -0.1 & $436.7 \pm 11$ & -22.1 \\
\hline Exp. ${ }^{a}$ & & 0.1 & 860.8 & & 561.0 & \\
\hline Exp. ${ }^{b}$ & & 0.1 & - & - & 554.2 & \\
\hline Exp.c & & 0.1 & 862.9 & & 559.5 & \\
\hline Ch-AUA & 313 & $0.1 \pm 0.3$ & $863.0 \pm 0.3$ & 2.0 & $445 \pm 30$ & -6.1 \\
\hline Exp. $^{a}$ & & 0.1 & 846.1 & & 474.0 & \\
\hline Flexible & 322 & 0.1 & 837.5 & -0.3 & $300.7 \pm 11.4$ & -30.0 \\
\hline Exp. ${ }^{\mathrm{c}}$ & & 0.1 & 840.2 & & 427.3 & \\
\hline Ch-AUA & 333 & $0.1 \pm 0.3$ & $843.4 \pm 0.1$ & 2.0 & $380 \pm 28$ & -2.6 \\
\hline Exp. $^{a}$ & & 0.1 & 827.5 & & 390.0 & \\
\hline Ch-AUA & 353 & $0.1 \pm 0.3$ & $823.5 \pm 0.3$ & 1.9a $(-0.8 c)$ & $301 \pm 25$ & -20.0 \\
\hline Flexible & 350 & 0.1 & 805.2 & -0.4 & $250.1 \pm 11.9$ & -34.0 \\
\hline Exp. ${ }^{a}$ & 353 & 0.1 & 808.3 & & 376.0 & \\
\hline Exp. ${ }^{\mathrm{c}}$ & 353 & 0.1 & 830.0 & & 384.5 & \\
\hline Ch-AUA & 363 & $0.1 \pm 0.4$ & $813.4 \pm 0.7$ & 1.9 & $283 \pm 20$ & -6.0 \\
\hline Exp. ${ }^{a}$ & 363 & 0.1 & 798.7 & & 301.0 & \\
\hline Ch-AUA & 333 & $40.0 \pm$ & $875.24 \pm 0.5$ & 1.8 & $510.5 \pm 60$ & 0.5 \\
\hline Exp..$^{a}$ & & 40.0 & 859.8 & & 513.0 & \\
\hline Ch-AUA & & $100.0 \pm$ & $909.0 \pm 0.7$ & --- & $633 \pm 50$ & -14.6 \\
\hline Exp. $^{a}$ & & 100.0 & --- & & 741.0 & \\
\hline Ch-AUA & & $250.0 \pm$ & $948.5 \pm 0.5$ & & $1372.1 \pm 80$ & \\
\hline Ch-AUA & & $500.0 \pm$ & $1019.4 \pm 0.4$ & & $4781.6 \pm 120$ & \\
\hline \multirow[t]{2}{*}{ AAD (\%) } & & Ch-AUA & & 1.9 & & 9.05 \\
\hline & & Flexible & & 0.3 & & 28.7 \\
\hline
\end{tabular}


o-xylene

\begin{tabular}{|c|c|c|c|c|c|c|}
\hline Ch-AUA & 273 & 0.1 & $900.0 \pm 0.2$ & & $988.0 \pm 28$ & -8.8 \\
\hline AUA & & 0.2 & $904.9 \pm 0.3$ & & $871.0 \pm 57$ & -19.6 \\
\hline Corr.d & & 0.1 & --- & & 1084 & \\
\hline Ch-AUA & 298 & 0.1 & $878.9 \pm 0.2$ & 0.6 & $736 \pm 59$ & -2.9 \\
\hline AUA & & 0.3 & $882.3 \pm 0.3$ & 0.9 & $675 \pm 40$ & -10.9 \\
\hline OPLS & & 0.1 & $875.8 \pm 8.7$ & 0.2 & $767 \pm 38$ & 1.2 \\
\hline Exp. $^{\text {a }}$ & & 0.1 & 874.0 & & 758.0 & \\
\hline Exp. $^{\text {e }}$ & & 0.1 & 876.5 & & 751.0 & \\
\hline Ch-AUA & 323.15 & $0.2 \pm 0.2$ & $855.8 \pm 04$ & 0.3 & $472 \pm 32$ & -16.1 \\
\hline AUA & & $0.4 \pm 0.1$ & 858.6.1 \pm 0.4 & 0.7 & $480 \pm 40$ & -14.6 \\
\hline OPLS & & 0.1 & $853.1 \pm 9.0$ & 0.0 & $535 \pm 27$ & -4.8 \\
\hline Exp. $^{\text {a }}$ & & 0.1 & 852.9 & & 562.0 & \\
\hline Exp. $^{\text {e }}$ & & 0.1 & 854.9 & & 560.0 & \\
\hline Ch-AUA & 348.15 & $0.1 \pm 0.4$ & $833.4 \pm 0.2$ & 0.2 & $408 \pm 31$ & -5.5 \\
\hline AUA & & $0.1 \pm 0.5$ & $835.5 \pm 0.4$ & 0.4 & $397 \pm 38$ & -8.1 \\
\hline OPLS & & 0.1 & $828.6 \pm 8.5$ & 0.4 & $458 \pm 23$ & 6.0 \\
\hline Exp. $^{\text {a }}$ & & 0.1 & 831.9 & & 432.0 & \\
\hline Exp. $^{\text {e }}$ & & 0.1 & 833.3 & & 445.0 & \\
\hline Ch-AUA & 373.15 & $0.4 \pm 0.2$ & $809.9 \pm 0.2$ & & $325 \pm 30$ & -5.9 \\
\hline Ch-AUA & & $0.1 \pm 0.1$ & $811.0 \pm 0.4$ & 3.1 & $292.0 \pm 13$ & -15.5 \\
\hline Corr. $^{\mathrm{d}}$ & & 0.1 & --- & & 345.0 & \\
\hline Ch-AUA & 323.15 & $40 \pm 0.7$ & $884.0 \pm 0.2$ & 0.4 & $741.0 \pm 37$ & -6.5 \\
\hline AUA & & $40.1 \pm 0.4$ & $887.5 \pm 0.1$ & 0.8 & $657.0 \pm 63$ & -17.2 \\
\hline OPLS & & 40.0 & $882.5 \pm 9.0$ & 0.2 & $820.0 \pm 41$ & 3.4 \\
\hline Exp. $^{\text {a }}$ & & 40.0 & 880.4 & & 793.0 & \\
\hline Corr. $^{\mathrm{d}}$ & & 40.0 & 882.4 & & 756.0 & \\
\hline Ch-AUA & & $100.0 \pm 0.7$ & $915.0 \pm 0.2$ & 0.6 & $1098 \pm 53$ & -5.7 \\
\hline AUA & & $100.0 \pm 0.1$ & $919.4 \pm 0.73$ & 1.1 & $1012 \pm 125$ & -13.1 \\
\hline OPLS & & 100.0 & $914.3 \pm 8.0$ & 0.5 & $1200 \pm 60$ & 3.0 \\
\hline Ch-AUA & & $250.0 \pm 0.7$ & $969.3 \pm 0.2$ & & $2173 \pm 150$ & \\
\hline Ch-AUA & & $500.0 \pm 0.7$ & $1026.6 \pm 0.2$ & & $14818 \pm 103$ & \\
\hline
\end{tabular}




\begin{tabular}{|c|c|c|c|c|c|c|}
\hline \multirow[t]{3}{*}{ AAD (\%) } & & ch-AUA & \multicolumn{3}{|c|}{0.4} & 7.3 \\
\hline & & \multicolumn{2}{|l|}{ AUA } & \multicolumn{2}{|l|}{0.8} & 13.9 \\
\hline & & \multicolumn{2}{|l|}{ OPLS } & \multicolumn{2}{|l|}{0.3} & 3.7 \\
\hline & & & \multicolumn{4}{|l|}{ m-xylene } \\
\hline Ch-AUA & 273 & 0.1 & $890.0 \pm 0.2$ & & $793.3 \pm 159$ & -0.2 \\
\hline AUA & & 0.2 & $871.0 \pm 0.3$ & & $454.0 \pm 55$ & -42.9 \\
\hline Corr. $^{\mathrm{d}}$ & & 0.1 & --- & & 795 & \\
\hline Ch-AUA & 298 & 0.1 & $868.0 \pm 0.3$ & 1.1 & $564 \pm 86$ & -2.8 \\
\hline AUA & & 0.1 & $870.8 \pm 0.3$ & 1.4 & $476 \pm 130$ & -17.9 \\
\hline OPLS & & 0.1 & $858.0 \pm 9.0$ & -0.1 & $532 \pm 26$ & -8.3 \\
\hline Exp. $^{a}$ & & 0.1 & 858.8 & & 580.0 & \\
\hline Exp. $^{\text {e }}$ & & 0.1 & 860.1 & & 526.0 & \\
\hline Ch-AUA & 323.15 & $-0.1 \pm 0.2$ & $845.8 \pm 01$ & 1.0 & $420.3 \pm 48$ & -6.3 \\
\hline AUA & & $0.4 \pm 0.1$ & $848.0 \pm 0.5$ & 1.3 & $363 \pm 32$ & -19.0 \\
\hline OPLS & & 0.1 & $835.7 \pm 9.0$ & -0.2 & $420 \pm 21$ & -6.3 \\
\hline Exp. $^{\text {a }}$ & & 0.1 & 837.1 & & 448.0 & \\
\hline Exp. $^{\text {e }}$ & & 0.1 & 839.4 & & 434.0 & \\
\hline Ch-AUA & 348.15 & $-0.3 \pm 1.9$ & $823.4 \pm 0.3$ & 0.9 & $332.9 \pm 45$ & -6.0 \\
\hline AUA & & $-0.1 \pm 0.3$ & $824.1 \pm 5.4$ & 1.1 & $316 \pm 43$ & -10.7 \\
\hline OPLS & & 0.1 & $810.9 \pm 9.0$ & -0.6 & $335 \pm 17$ & -5.4 \\
\hline Exp. $^{a}$ & & 0.1 & 815.4 & & 354.0 & \\
\hline Exp. $^{\text {e }}$ & & 0.1 & 817.6 & & 350.0 & \\
\hline Ch-AUA & 373.15 & $0.7 \pm 0.3$ & $800.1 \pm 0.4$ & & $279.5 \pm 40$ & -3.4 \\
\hline AUA & & $0.8 \pm 0.3$ & $800.0 \pm 0.3$ & & $267.0 \pm 15$ & -7.6 \\
\hline Corr. $^{\mathrm{d}}$ & & 0.1 & --- & & 289.0 & \\
\hline Ch-AUA & 323.15 & $40 \pm 0.4$ & $873.9 \pm 0.4$ & 0.9 & $570.0 \pm 40$ & -4.5 \\
\hline AUA & & $40 \pm 0.2$ & $876.6 \pm 0.6$ & 1.2 & $549.0 \pm 99$ & -8 \\
\hline OPLS & & 40.0 & $865.4 \pm 9.0$ & -0.1 & $592.0 \pm 30$ & -0.8 \\
\hline Exp. $^{a}$ & & 40.0 & 866.1 & & 597.0 & \\
\hline Corr. $^{\mathrm{d}}$ & & 40.0 & 869.9 & & 594.0 & \\
\hline Ch-AUA & & $100.0 \pm 0.7$ & $906.2 \pm 0.2$ & 0.7 & $803 \pm 93$ & -5.9 \\
\hline AUA & & $100.0 \pm 0.1$ & $909.3 \pm 0.6$ & 1.0 & $771 \pm 100$ & -9.6 \\
\hline
\end{tabular}




\begin{tabular}{|c|c|c|c|c|c|c|}
\hline OPLS & & 100.0 & $898.6 \pm 9.0$ & 0.5 & $870.0 \pm 44$ & 2.0 \\
\hline Ch-AUA & & $250.0 \pm 0.7$ & $959.5 \pm 0.2$ & & $1473 \pm 160$ & \\
\hline Ch-AUA & & $500.0 \pm 0.7$ & $1017.3 \pm 0.2$ & & $5168 \pm 880$ & \\
\hline \multirow[t]{4}{*}{ AAD (\%) } & & ch-AUA & & 1.0 & & 4.3 \\
\hline & & AUA & & 1.2 & & 16.5 \\
\hline & & OPLS & & 0.2 & & 4.5 \\
\hline & & & p-xylene & & & \\
\hline Ch-AUA & 298 & 0.1 & $865.0 \pm 0.2$ & 0.2 & $690 \pm 36$ & 11.5 \\
\hline AUA & & 0.1 & $868.2 \pm 0.3$ & 1.6 & $565 \pm 130$ & -8.7 \\
\hline OPLS & & 0.1 & $854.0 \pm 9.0$ & -0.1 & $609 \pm 30$ & -1.6 \\
\hline Exp. $^{a}$ & & 0.1 & 854.9 & & 619.0 & \\
\hline Exp. $^{\text {e }}$ & & 0.1 & 855.8 & & 584.0 & \\
\hline Ch-AUA & 323.15 & $-0.2 \pm 0.2$ & $842.5 \pm 0.2$ & 1.1 & $470.7 \pm 26$ & -0.3 \\
\hline AUA & & $-0.03 \pm 0.4$ & $845.5 \pm 0.3$ & 1.4 & $462 \pm 38$ & -2.1 \\
\hline OPLS & & 0.1 & $831.5 \pm 9.0$ & -0.3 & $467 \pm 23$ & -1.1 \\
\hline Exp. $^{\text {a }}$ & & 0.1 & 833.7 & & 472.0 & \\
\hline Exp. $^{\mathrm{e}}$ & & 0.1 & 834.9 & & 452.0 & \\
\hline Ch-AUA & 348.15 & $0.1 \pm 0.4$ & $819.5 \pm 0.3$ & 0.8 & $355 \pm 11$ & -3.3 \\
\hline AUA & & $0.1 \pm 0.5$ & $822.4 \pm 0.5$ & 1.2 & $292 \pm 36$ & -20.4 \\
\hline OPLS & & 0.1 & $807.3 \pm 9.0$ & -0.7 & $385 \pm 19$ & 4.9 \\
\hline Exp. $^{\text {a }}$ & & 0.1 & 812.6 & & 367.0 & \\
\hline Exp. $^{\mathrm{e}}$ & & 0.1 & 812.9 & & 366.0 & \\
\hline Ch-AUA & 373.15 & $0.2 \pm 0.1$ & $795.6 \pm 0.3$ & & $290.7 \pm 17$ & 0.2 \\
\hline AUA & & $0.9 \pm 0.4$ & $798.3 \pm 0.7$ & & $254.0 \pm 31$ & -12.4 \\
\hline Corr.d & & 0.1 & --- & & 290.0 & \\
\hline Ch-AUA & 323.15 & $40 \pm 0.1$ & $871.0 \pm 0.2$ & 0.9 & $613.0 \pm 32$ & -0.8 \\
\hline AUA & & $40 \pm 0.2$ & $876.1 \pm 0.3$ & 1.5 & $523.0 \pm 56$ & -15.4 \\
\hline OPLS & & 40.0 & $862.1 \pm 9.0$ & -0.1 & $599.0 \pm 30$ & -3.1 \\
\hline Exp. $^{a}$ & & 40.0 & 863.0 & & 618.0 & \\
\hline Corr. $^{\mathrm{d}}$ & & 40.0 & 865.3 & & 615.0 & \\
\hline Ch-AUA & & $100.0 \pm 0.2$ & $903.2 \pm 0.2$ & 1.3 & $889 \pm 58$ & -2.2 \\
\hline AUA & & $100.0 \pm 0.1$ & $908.6 \pm 0.3$ & 1.9 & $746 \pm 100$ & -17.9 \\
\hline
\end{tabular}




$\begin{array}{llllll}\text { OPLS } & 100.0 & 895.7 \pm 9.0 & 0.5 & 920.0 \pm 46 & 1.2 \\ \text { Exp. }^{\mathrm{a}} & 100.0 & 891.6 & & 909.0 & \\ \text { Corr. }^{\mathrm{d}} & 100.0 & 898.0 & & 898.0 \\ \text { Ch-AUA } & 250.0 \pm 0.7 & 957.6 \pm 0.2 & & 2060 \pm 170 & \\ \text { Ch-AUA } & 500.0 \pm 0.7 & 1015.2 \pm 0.2 & & 8918 \pm 750 & \\ \text { AAD (\%) } & \text { ch-AUA } & & 1.0 & & 3.0 \\ & \text { AUA } & & 1.5 & & 12.8 \\ & \text { OPLS } & & 0.3 & & 2.4\end{array}$


Experimental data from Krall et al. ${ }^{\text {Error! Bookmark not defined. d }}$ Correlation from Assael et al. ${ }^{\text {Error! Bookmark }}$ not defined. e Handbook of physical data of fluids. ${ }^{\text {Error! Bookmark not defined. }}$ 